\title{
MATRIX DIFFERENTIAL SYSTEMS WITH A PARAMETER IN THE BOUNDARY CONDITIONS AND RELATED VIBRATION PROBLEMS*
}

\author{
BY \\ JULIĀN ADEM \\ Instituto de Geofísica, Universidad Nacional de M exico \\ and \\ Instituto Nacional de la Investigación Científica \\ México, D.F., (MEXICO)
}

In a series of papers by Moshinsky [1, 3] and Adem and Moshinsky [2] the description of some physical processes has been given by a column matrix or vector instead of a single function, achieving a simplification in the mathematical solution of such processes and making it possible to solve a variety of problems. In the examples considered in [2, 3], for example, the above formulation leads to a matrix eigenvalue problem which is a generalization of the Sturm-Liouville problem and that can be treated along similar lines.

In this paper, by describing a vibratory system in the aforementioned way, we are led to a more general eigenvalue problem that contains a parameter both in the differential equations and in the boundary conditions. This problem can be considered as a generalization of the corresponding scalar case, examples of which have been considered recently by Bauer [4] and Morgan [5].

In the first part of this paper, we give examples of vibratory systems that give rise to this new type of eigenvalue problem.

In the second part of this paper, we formulate and discuss the general matrix differential system and obtain some properties of the eigenvalues and eigen column matrices.

I. Vibrating string with a concentrated mass at a point. Consider a string with a concentrated mass at a point which separates two regions of different density and length of which the string is composed. The string is fixed at the ends. We wish to find the transverse displacements of the string if it is displaced initially into a position and released from rest at this position with no external forces acting.

Let the length of the string be $L_{1}+L_{2}$, and $M$ the concentrated mass at a distance $L_{1}$ from the left end point of the string. We shall designate I and II as the regions to the left and right of the concentrated mass respectively, and $Y_{1}$ and $Y_{2}$ as the corresponding displacements. Region I has a density $\rho_{1}$, and a length $L_{1}$, while II has a density $\rho_{2}$ and a length $L_{2}$. The space-independent variable shall be called $x_{1}$ in $\mathrm{I}$ and $x_{2}$ in II; $x_{1}$ increases from left to right, while $x_{2}$ increases from right to left, so that at the end points we have $x_{1}=0, x_{2}=0$ and at the point with the concentrated mass, $x_{1}=L_{1}, x_{2}=L_{2}$.

Considering that in both regions the equation of a vibrating string applies and considering boundary and initial conditions we obtain the following differential equations and boundary conditions:

${ }^{*}$ Received June 11, 1958. Numbers in square brackets refer to the bibliography at the end of the paper. 


$$
\begin{gathered}
\frac{\partial^{2} Y_{i}}{\partial x_{i}^{2}}=\frac{1}{C_{i}^{2}} \frac{\partial^{2} Y_{i}}{\partial t^{2}} \quad(i=1,2), \\
Y_{1}(0, t)=Y_{2}(0, t)=0 \\
Y_{1}\left(L_{1}, t\right)=Y_{2}\left(L_{2}, t\right) \\
\left(\frac{\partial Y_{1}}{\partial x_{1}}\right)_{x_{1}-L_{1}}+\left(\frac{\partial Y_{2}}{\partial x_{2}}\right)_{x_{2}=L_{2}}=-\frac{M}{T}\left(\frac{\partial^{2} Y_{1}}{\partial t^{2}}\right)_{x_{1}=L_{1}} \\
Y_{1}\left(x_{1}, 0\right)=F_{1}\left(x_{1}\right) ; \quad Y_{2}\left(x_{2}, 0\right)=F_{2}\left(x_{2}\right), \\
\left(\frac{\partial Y_{1}}{\partial t}\right)_{t=0}=\left(\frac{\partial Y_{2}}{\partial t}\right)_{t=0}=0,
\end{gathered}
$$

where $C_{i}^{2}=T / \rho_{i}(i=1,2) ; T$ is the tension on the string; $F_{1}\left(x_{1}\right)$ and $F_{2}\left(x_{2}\right)$ are the initial displacements prescribed at regions I and II respectively.

Applying the change of independent variables

$$
x_{i}=L_{i} x_{i}^{\prime} \quad(i=1,2),
$$

we have that now the range of $x_{1}^{\prime}$ is the same as that of $x_{2}^{\prime}$ and we can therefore put $x_{1}^{\prime}=x_{2}^{\prime}=x$.

Problem (1), (2), (3), (4), becomes:

$$
\begin{gathered}
\frac{1}{L_{i}^{2}} \frac{\partial^{2} y_{i}}{\partial x^{2}}=\frac{1}{C_{i}^{2}} \frac{\partial^{2} y_{i}}{\partial t^{2}} \text { for } 0<x<1, \\
y_{1}(0, t)=y_{2}(0, t)=0 \\
y_{1}(1, t)=y_{2}(1, t) \\
\frac{1}{L_{1}}\left(\frac{\partial y_{1}}{\partial x}\right)_{x=1}+\frac{1}{L_{2}}\left(\frac{\partial y_{2}}{\partial x}\right)_{x=1}=-\frac{M}{T}\left(\frac{\partial^{2} y_{1}}{\partial t^{2}}\right)_{x=1} \\
y_{1}(x, 0)=f_{1}(x) ; \quad y_{2}(x, 0)=f_{2}(x) \\
\left(\partial y_{1} / \partial t\right)_{t=0}=\left(\partial y_{2} / \partial t\right)_{t=0},
\end{gathered}
$$

where

$$
x=x_{i} / L_{i}, \quad y_{i}(x, t)=Y_{i}\left(x_{i}, t\right)
$$

and

$$
f_{i}(x)=F_{i}\left(x_{i}\right)
$$

Let

$$
y_{i}(x, t)=y_{i}(x) \cos \omega t
$$

then problem $\left(1^{\prime}\right),\left(2^{\prime}\right)$ becomes:

$$
\left.\begin{array}{c}
\frac{1}{L_{i}^{2}} \frac{d^{2} y_{i}}{d x^{2}}+\frac{\omega^{2}}{C_{i}^{2}} y_{i}=0 \quad(i=1,2) \\
y_{1}(0)=y_{2}(0)=0 \\
y_{1}(1)=y_{2}(1) \\
\left(\frac{1}{L_{1}} \frac{d y_{1}}{d x}+\frac{1}{L_{2}} \frac{d y_{2}}{d x}\right)_{x=1}-\frac{M}{T} \omega^{2} y_{1}(1)=0
\end{array}\right\} .
$$


The solution of Eq. (5) is

$$
y_{i}(x)=A_{i} \cos \left(\omega L_{i} x / C_{i}\right)+B_{i} \sin \left(\omega L_{i} x / C_{i}\right),
$$

where $A_{i}, B_{i}$ and $\omega$ are going to be determined from the boundary conditions.

From conditions (6) it follows that $A_{i}=0$.

From conditions ( 7 ) we get the frequency equation:

$$
\left.\begin{array}{c}
\left(1 / C_{2}\right) \sin \left(\omega L_{1} / C_{1}\right) \cos \left(\omega L_{2} / C_{2}\right)+\left(1 / C_{1}\right) \cos \left(\omega L_{1} / C_{1}\right) \sin \left(\omega L_{2} / C_{2}\right) \\
-(M \omega / T) \sin \left(\omega L_{1} / C_{1}\right) \sin \left(\omega L_{2} / C_{2}\right)=0
\end{array}\right\}
$$

whose roots are real and form an infinite denumerable set. Let the positive roots of Eq. (8) be $\omega_{1}, \omega_{2}, \cdots, \omega_{n}, \cdots$. They are the eigenvalues of the problem (5), (6), (7).

To each eigenvalue there corresponds a solution:

$$
\left.\begin{array}{l}
y_{1 i}(x)=\sin \left(\omega_{i} L_{1} x / C_{1}\right) \\
y_{2 j}(x)=\frac{\sin \left(\omega_{i} L_{1} / C_{1}\right)}{\sin \left(\omega_{i} L_{2} / C_{2}\right)} \sin \left(\omega_{i} L_{2} x / C_{2}\right)
\end{array}\right\}
$$

where we have used the notation $y_{1 j}(x), y_{2 i}(x)$ instead of $y_{1}(x), y_{2}(x)$ to distinguish that it is a solution corresponding to the $\omega_{i}$ eigenvalue. $y_{1 i}(x)$ and $y_{2 i}(x)$ can be considered as the components of a column matrix (vector) which will be denoted by $\mathrm{y}_{i}(x)$ and called eigen column matrix:

$$
\mathbf{y}_{i}(x)=\left[\begin{array}{l}
y_{1 j}(x) \\
y_{2 i}(x)
\end{array}\right]
$$

Proceeding as usual, we assume as solution of our problem an infinite series of the type:

$$
\mathrm{y}(x, t)=\sum_{i=1}^{\infty} B_{i} \mathbf{y}_{i}(x) \cos \omega_{j} t .
$$

This series is a solution of $\left(1^{\prime}\right),\left(2^{\prime}\right),\left(4^{\prime}\right)$ provided it is convergent and admits two successive term by term differentiations with respect to $t$ and $x$. Assuming that this condition is satisfied, there remains, then, to determine the constants $B_{i}$, so that $\left(3^{\prime}\right)$ is satisfied:

$$
\mathbf{f}(x) \equiv\left[\begin{array}{l}
f_{1}(x) \\
f_{2}(x)
\end{array}\right]=\sum_{i=1}^{\infty} B_{i}\left[\begin{array}{l}
y_{1 i}(x) \\
y_{2 i}(x)
\end{array}\right] \equiv \sum_{i=1}^{\infty} B_{i} \mathbf{y}_{i}(x) .
$$

Assuming the validity of expansion (10) the coefficients $B_{i}$ can easily be determined and for it we shall establish first the orthogonality relation of the eigen column matrices corresponding to the problem (5), (6), (7).

Since $y_{k}(x)$ and $y_{p}(x)$ are solutions of (5) corresponding to $\omega_{k}$ and $\omega_{p}$ respectively, the following relation is satisfied:

$$
\sum_{i=1}^{2} \frac{1}{L_{i}}\left(y_{i k} \frac{d^{2} y_{i p}}{d x^{2}}-\frac{d^{2} y_{i k}}{d x^{2}} y_{i p}\right)=\sum_{i=1}^{2} \frac{L_{i}}{C_{i}^{2}}\left(\omega_{k}^{2}-\omega_{p}^{2}\right) y_{i k} y_{i p} .
$$

Therefore

$$
\sum_{i=1}^{2} \frac{1}{L_{i}}\left[y_{i k} \frac{d y_{i p}}{d x}-y_{i p} \frac{d y_{i k}}{d x}\right]_{0}^{1}=\left(\omega_{k}^{2}-\omega_{p}^{2}\right) \int_{0}^{1} \sum_{i=1}^{2} \frac{L_{i}}{C_{i}^{2}} y_{i k} y_{i p} d x
$$


From boundary conditions (6) and (7) it follows that

$$
\sum_{i=1}^{2} \int_{0}^{1} \frac{L_{i}}{C_{i}^{2}} y_{i k}(x) y_{i p}(x) d x+\frac{M}{T} y_{1 k}(1) y_{1 p}(1)=0
$$

or with matrix notation:

$$
\int_{0}^{1} \mathbf{y}_{k}^{\prime}(x) \mathbf{H y}_{p}(x) d x+\mathbf{y}_{k}^{\prime}(1) \mathrm{Jy}_{p}(1)=0
$$

where

$$
\mathbf{H}=\left(\begin{array}{cc}
L_{1} / C_{1}^{2} & 0 \\
0 & L_{2} / C_{2}^{2}
\end{array}\right] ; \quad \mathrm{J}=\left[\begin{array}{cc}
M / T & 0 \\
0 & 0
\end{array}\right)
$$

and $\mathbf{y}_{k}^{\prime}(x)$ denotes the transposed of $\mathbf{y}_{k}(x)$.

This is an orthogonality condition for the eigen column matrices of problem (5), (6), (7).

Assuming that the series (10) can be integrated term by term after being multiplied by $\mathbf{y}_{i}^{\prime}(x) \mathrm{H}$, we can now, using (11), determine the coefficients $B_{i}$ in (10), which are given by

$$
B_{i}=\frac{\int_{0}^{1}\left[\left(L_{1} / C_{1}^{2}\right) f_{1}(x) y_{1 i}(x)+\left(L_{2} / C_{2}^{2}\right) f_{2}(x) y_{2 j}(x)\right] d x+(M / T) f_{1}(1) y_{1 i}(1)}{\int_{0}^{1}\left[\left(L_{1} / C_{1}^{2}\right) y_{1 i}^{2}(x)+\left(L_{2} / C_{2}^{2}\right) y_{2 i}^{2}(x)\right] d x+(M / T) y_{1 j}^{2}(x)}
$$

or more briefly with matrix notation

$$
B_{i}=\frac{\int_{0}^{1} \mathbf{y}_{i}^{\prime}(x) \mathbf{H f}(x) d x+\mathbf{f}^{\prime}(1) \mathrm{Jy}_{i}(1)}{\int_{0}^{1} \mathbf{y}_{i}^{\prime}(x) \mathbf{H y}_{i}(x) d x+\mathbf{y}_{i}^{\prime} \mathrm{Jy}_{i}(1)} .
$$

Substituting (9) into (12) we obtain

$$
\begin{aligned}
\frac{1}{2}\left[\frac{L_{1}}{C_{1}^{2}}+\sin ^{2} \frac{\omega_{i} L_{1}}{C_{1}}\right]\left[\frac{L_{2}}{C_{2}^{2} \sin ^{2}\left(\omega_{i} L_{2} / C_{2}\right)}+\frac{M}{T}\right] B_{i} \\
=\frac{L_{1}}{C_{1}^{2}} \int_{0}^{1} f_{1}(x) \sin \frac{\omega_{i} L_{1} x}{C_{1}} d x+\frac{L_{2} \sin \left(\omega_{j} L_{1} / C_{1}\right)}{C_{2}^{2} \sin \left(\omega_{i} L_{2} / C_{2}\right)} \int_{0}^{1} f_{2}(x) \sin \frac{\omega_{i} L_{2} x}{C_{2}} d x \\
+\frac{M}{T} f_{1}(1) \sin \frac{\omega_{i} L_{1}}{C_{1}},
\end{aligned}
$$

and the formal solution to the problem (1), (2), (3), (4), is:

$$
\left[\begin{array}{l}
Y_{1}\left(x_{1}, t\right) \\
Y_{2}\left(x_{2}, t\right)
\end{array}\right]=\sum_{i=1}^{\infty} B_{i}\left(\begin{array}{c}
\sin \left(\omega_{i} x_{1} / C_{1}\right) \\
\frac{\sin \left(\omega_{i} L_{1} / C_{1}\right)}{\sin \left(\omega_{i} L_{2} / C_{2}\right)} \sin \left(\omega_{i} x_{2} / C_{2}\right)
\end{array}\right) \cos \omega_{i} t
$$

where $\omega_{i}$ are the positive roots of Eq. (8) and the $B_{i}$ 's are given by (13).

II. Solution of other vibrating systems. The above method can be applied to the case of a string with $n$ concentrated masses $M_{1}, M_{2}, \cdots, M_{n}$ which separate $n+1$ 
regions of different density $\rho_{1}, \rho_{2}, \cdots, \rho_{n}$; and different length $L_{1}, L_{2}, \cdots, L_{n}$. In this case we describe the system by $n+1$ functions $Y_{1}, Y_{2}, \cdots$, which by a change of variable is always possible to have in the same domain, for example, $0 \leq x \leq 1, t \geq 0$. We call the $n+1$ regions from left to right, I, II, III, $\cdots$ and, for example, in the regions labeled by an odd number let the space variable increase from left to right, while in those labeled by an even number let it increase from right to left. We are led to a system of $n+1$ differential equations of the type $\left(1^{\prime}\right)$ with boundary conditions similar to conditions $\left(2^{\prime}\right),\left(3^{\prime}\right),\left(4^{\prime}\right)$. In fact, we are led to a matrix differential system which is a particular case of the one discussed in the next section

Since the solution of the mathematical problem corresponding to a vibrating string also solves analogous problems of torsional vibrations of shafts of circular section and longitudinal vibrations of bars, the above method yields exact solutions (without neglecting the mass of the bars, as is usual in Theory of Vibrations) of such systems when they have several concentrated masses, which separate bars of different elasticity, section and length

III. General matrix differential system. The problem (5), (6), (7) as well as those that arise in the examples of Sec. II, are particular cases of the system formed by the general self-adjoint matrix differential equation [6]

$$
L(\mathbf{y}) \equiv \frac{d}{d x}\left(\mathbf{P} \frac{d \mathbf{y}}{d x}\right)+\mathbf{Q} \frac{d \mathbf{y}}{d x}+\left(\frac{1}{2} \frac{d \mathbf{Q}}{d x}+\mathbf{R}\right) \mathbf{y}=\lambda \mathbf{W y}
$$

and boundary conditions of the type

$$
\left.\begin{array}{l}
(\mathbf{A}+\lambda \mathbf{B}) \mathbf{y}(0)+\mathbf{C}(d \mathbf{y} / d x)_{x=0}=0 \\
(\mathbf{D}+\lambda \mathbf{E}) \mathbf{y}(1)+\mathbf{F}(d \mathbf{y} / d x)_{x=1}=0
\end{array}\right\}
$$

where $\mathbf{P}, \mathbf{R}$ and $\mathbf{W}$ are $n \times n$ symmetric matrices, $\mathbf{Q} n \times n$ antisymmetric matrix; $\mathbf{P}$, $\mathrm{R}, \mathrm{W}, \mathrm{Q}$ are real functions of $x$; the unknown $\mathrm{y}$ is a column matrix of $n$ components; $\lambda$ is a scalar parameter; A, B, C, D, E, F, are $n \times n$ constant real matrices. We assume that $\mathbf{P}, \mathbf{R}, \mathbf{W}, \mathbf{Q}, d \mathbf{P} / d x, d \mathbf{Q} / d x$ are continuous and that the determinant of $\mathbf{P}$ is different from zero in $0<x<1$.

From (15) we obtain

$$
\begin{aligned}
\int_{0}^{1}\left[\mathbf{y}_{i}^{\prime} L\left(\mathbf{y}_{i}\right)\right. & \left.-L^{\prime}\left(\mathbf{y}_{i}\right) \mathbf{y}_{i}\right] d x \\
& \equiv\left[\mathbf{y}_{i}^{\prime} \mathbf{P}\left(d \mathbf{y}_{i} / d x\right)-\left(d \mathbf{y}_{i}^{\prime} / d x\right) \mathbf{P} \mathbf{y}_{i}+\mathbf{y}_{i}^{\prime} \mathbf{Q} \mathbf{y}_{i}\right]_{0}^{1}=\left(\lambda_{i}-\lambda_{i}\right) \int_{0}^{1} \mathbf{y}_{i}^{\prime} \mathbf{W y}_{i} d x,
\end{aligned}
$$

where $y_{i}$ and $y_{i}$ are two different solutions (eigen column matrices) corresponding to the eigenvalues $\lambda_{i}$ and $\lambda_{i}$ respectively; and $y_{i}^{\prime}$ and $L^{\prime}\left(y_{i}\right)$ denote the transposed of $y_{i}$ and $L\left(y_{i}\right)$ respectively (i.e., they are row matrices of $n$ elements).

The problem that we are going to deal with is such that by substituting the boundary conditions (15) into (16) we obtain a relation of the type:

$$
\left[\mathbf{y}_{i}, \mathbf{y}_{i}\right] \equiv \int_{0}^{1} \mathbf{y}_{i}^{\prime}(x) \mathbf{W}(x) \mathbf{y}_{j}(x) d x+\mathbf{y}_{i}^{\prime}(0) \mathbf{M} \mathbf{y}_{i}(0)+\mathbf{y}_{i}^{\prime}(1) \mathbf{N y}_{i}(1)=0,
$$

where $\mathbf{M}$ and $\mathbf{N}$ are constant $n \times n$ matrices. Relation (17) can be considered as an orthogonality relation that the eigen column matrices of the problem (14), (15) satisfy. 
We are more familiar with orthogonality relations in which the last two terms of the left member of Eq. (17) are zero. Such is the case in the self-adjoint problem of the type (14), (15) but in which the parameter $\lambda$ does not appear in the boundary conditions (i.e., $B=E=0$ ). This problem has been extensively studied by G. D. Birkhoff and R. E. Langer [7]; and also by Moshinsky and the author in connection with selfadjointness [6], and examples of it have been given in [2,3]. As far as the present writer is aware the problem (14), (15) has only been studied in the scalar case (i.e., when $n=1)$ and some papers by Bauer and Morgan have appeared recently dealing with it $[4,5]$.

Now that we have formulated the mathematical problem (14), (15) the next step would be to develop a theory dealing with properties of eigenvalues and eigen column matrices and with the possibility of expanding an arbitrary column matrix in terms of the eigen column matrices, as is needed for the applications, as, for example, in the problems mentioned in the above sections.

Proceeding as W F. Bauer does in the scalar case [4], we shall give the following definitions:

D.1. A column matrix $\mathbf{u}(x)$ shall be called $V$-column matrix if it is real, not identically zero, of class $C^{1}$, and satisfies the boundary conditions (15).

D.2. The inner product corresponding to the eigenvalue problem (14), (15) is defined as

$$
[\mathbf{h}, \mathbf{g}] \equiv \int_{0}^{1} \mathbf{h}^{\prime}(x) \mathbf{W}(x) \mathbf{g}(x) d x+\mathbf{h}^{\prime}(0) \mathbf{M g}(0)+\mathbf{h}^{\prime}(1) \mathbf{N g}(1),
$$

where $\mathbf{h}(x)$ and $\mathbf{g}(x)$ are column matrices whose components are bounded integrable functions.

D.3. The eigenvalue problem (14), (15) is normal if for every $V$-column matrix $\mathbf{u}(x)$ we have

$$
[\mathbf{u}, \mathbf{u}]>0 .
$$

The following results can be established:

T.1. A sufficient condition for the problem (14), (15) to be normal is that W be a non-zero diagonal matrix whose components are non-negative in the fundamental interval and $\mathbf{N}$ and $\mathbf{M}$ diagonal matrices whose components are non-negative.

T.2. If $\mathbf{W}, \mathbf{N}$ and $\mathbf{M}$ satisfy the same condition as in T.1., the eigenvalue problem (14), (15) has only real eigenvalues. The proof of T.2., is analogous to that employed in the scalar case [4].

T.3. If we define as Rayleigh's quotient $R(\mathrm{y})$ for any $V$-column matrix $\mathrm{y}(x)$

$$
R(\mathrm{y})=\frac{[L(\mathrm{y}), \mathrm{y}]}{[\mathbf{W} \mathbf{y}, \mathrm{y}]},
$$

where $L(\mathrm{y})$ stands for the left member of (14), from (14) it follows that $R\left(\mathrm{y}_{i}\right)=\lambda_{i}$, where $y_{i}$ is an eigen column matrix corresponding to the eigenvalue $\lambda_{i}$.

T.4. If $\mathbf{W}, \mathbf{N}$ and $\mathbf{M}$ satisfy the same conditions as in T.1., and $[L(\mathrm{y}), \mathrm{y}]>0$ for every $V$-column matrix then the eigenvalue problem (14), (15) has only positive eigenvalues. This result follows from T.3.

T.5. Corresponding to each real eigenvalue there is one or at the most, a finite number $h_{i}$ of linearly independent real eigen column matrices $y_{i}^{(\alpha)}(x), \alpha=1,2, \cdots, h_{i} .\left(h_{i} \leq 2 n\right)$. 
T.6. The eigen column matrices corresponding to different eigenvalues are orthogonal with respect to the inner product defined by (18). The eigen column matrices corresponding to the same eigenvalue can be normalized (we assume that the norm of all eigen column matrices is different from zero) and made mutually orthogonal, by the usual procedure [8] so that our set of eigen column matrices satisfies

$$
\left[y_{i}^{(\alpha)}, y_{i}^{(\beta)}\right]=\delta_{\alpha \beta} \delta_{i j}
$$

where

$$
\alpha=1,2, \cdots, h_{i} ; \quad \beta=1,2, \cdots, h_{i} ; \quad i, j=1,2, \cdots
$$

and $\delta_{\alpha \beta}$ is equal to 1 for $\alpha=\beta$ and equal to zero for $\alpha \neq \beta$.

T.7. If we assume the validity of an expansion of the type

$$
f(x)=\sum_{n=1}^{\infty} \sum_{\alpha=1}^{h_{n}} A_{n}^{(\alpha)} y_{n}^{(\alpha)}(x)
$$

where $\mathbf{f}(x)$ is a column matrix whose components are bounded integrable functions of $x$ [that satisfy certain conditions that ensure the possibility of expansion (20)], and if the series (20) can be integrated term by term after being multiplied by $\mathbf{y}_{m}^{\prime}(x) \mathbf{W}(x)$, it is possible, using (19), to obtain the coefficients $A_{m}^{(\beta)}$, which are given by

$$
A_{m}^{(\beta)}=\left[\mathbf{f}(x), \mathbf{y}_{m}^{(\beta)}\right] .
$$

The conditions under which the expansion (20) is possible as well as the existence of an infinite denumerable set of eigenvalues, and some properties of eigenvalues and eigen column matrices of the problem (14), (15) will be left unsettled.

\section{References}

1. Marcos Moshinsky, Phys. Rev. 81, 347 (1951)

2. Julián Adem and Marcos Moshinsky, Quart. Appl. Math. 9, 424 (1951)

3. Marcos Moshinsky, Bol. Soc. Mat. Mexicana 4, No. 1, 4, p. 1 (1947)

4. W. F. Bauer, Quart. Appl. Math. 11, 274, (1953); see also the references mentioned in this paper

5. G. W. Morgan, Quart. Appl. Math. 11, 157 (1953)

6. Julián Adem and Marcos Moshinsky, Bol. Soc. Mat. Mexicana 7, No. 3, 4, p. 1 (1950)

7. G. D. Birkhoff and R. E. Langer, Proc. Am. Acad. Arts Sci. 58, 51 (1923)

8. R. Courant and D. Hilbert, Methods of Mathematical Physics, 1st. English ed., Interscience Publishers, Inc., 1953, p. 50 